\title{
Expression of oxidative and nitrosative stress genes in the context of antidepressant treatment in CMS model of depression
}

Paulina Wigner1, Ewelina Synowiec1, Piotr Czarny2, Michal Bijak3, Pawel Jozwiak4, Janusz Szemraj2, Piotr Gruca5, Mariusz Papp5, Tomasz Sliwinski1.

1University of Lodz, Faculty of Biology and Environmental Protection, Laboratory of Medical Genetics, Lodz, Poland. 2Medical University of Lodz, Department of Medical Biochemistry, Lodz, Poland.

3University of Lodz, Faculty of Biology and Environmental Protection, Department of General Biochemistry, Lodz, Poland.

4University of Lodz, Faculty of Biology and Environmental Protection, Department of Cytobiochemistry, Lodz, Poland.

5 Institute of Pharmacology, Polish Academy of Sciences, Krakow, Poland.

Background

Recent human and animal studies indicate that oxidative and nitrosative stress (O\&NS) may play a role in the aetiology and pathogenesis of depression. This study investigates the effect of chronic administration of the serotonin-norepinephrine reuptake inhibitor, venlafaxine, on the expression and methylation status of SOD1, SOD2, GPx1, GPx4, CAT, NOS1 and NOS2 in the brain and peripheral mononuclear blood cells (PBMCs) of rats exposed to a chronic mild stress (CMS) model of depression.

Methods

Separate groups of animals were exposed to CMS for two or seven weeks; the second group received saline or venlafaxine (10 mg/kg/day, IP) for five weeks. After completion of both stress conditions and drug administration, the mRNA and protein expression of selected genes and the methylation status of their promoters, were measured in PBMCs and in brain structures (hippocampus, amygdala, hypothalamus, midbrain, cortex, basal ganglia) with the use of TaqMan Gene Expression Assay, Western blot and methylation-sensitive high-resolution melting techniques.

Results

CMS caused a decrease of sucrose consumption and this effect was normalized by venlafaxine. In PBMCs, SOD1, SOD2 and NOS2 mRNA expression changed only after venlafaxine administration. In brain, CAT, Gpx1, Gpx4 and NOS1 gene expression changed following CMS or venlafaxine exposure, most prominently in the hippocampus, midbrain and basal ganglia. CMS increased the methylation of the Gpx1 promoter in PBMCs, the second Gpx4 promoter in midbrain and basal ganglia, and SOD1 and SOD2 in hippocampus. The CMS animals treated with venlafaxine displayed a significantly higher CAT level in midbrain and cerebral cortex. CMS caused an elevation of Gpx4 in the hippocampus, which was lowered in cerebral cortex by venlafaxine.

\section{Conclusion}

The results indicate that CMS and venlafaxine administration affect the methylation of promoters of genes involved in O\&NS. They also indicate that peripheral and central tissue differ in their response to stress or antidepressant treatments. It is possible that that apart from DNA methylation, a crucial role in epigenetic regulation may be played by histone modifications or microRNA interference.

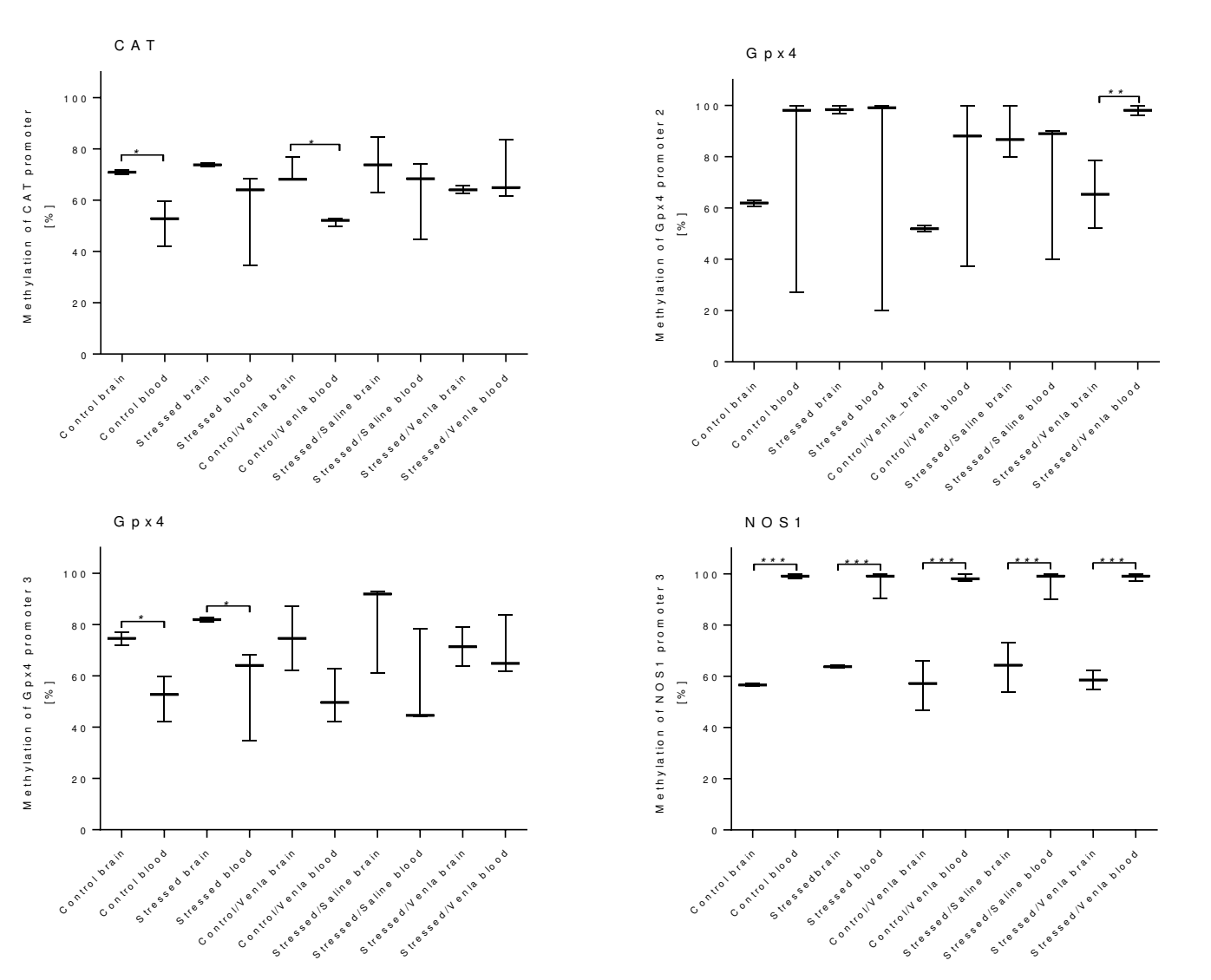

Differences in methylation level of CAT, Gpx4 promoter 2, Gpx4 promoter 3 and NOS1 promoter 3 between brain tissue and PBMCs of animals exposed to CMS for two weeks (Control, Stressed) and in animals exposed to CMS for seven weeks and administered vehicle $(1 \mathrm{~m} / \mathrm{kg})$ or venlafaxine $(10$ $\mathrm{mg} / \mathrm{kg}$ ) for five weeks (Control/Venla, Stressed/Saline, Stressed/Venla). The methylation level of all $\mathrm{mg} / \mathrm{kg}$ ) for five weeks (Contro/ $/$ enla, Stressed/Saline, Stressed/Venla). The methylation level of all were pooled and compared with the data detected in PBMCs. Data represent means \pm SEM. $N=6$. ${ }^{*} p<0.05,{ }^{* *} p<0.01,{ }^{* * *} p<0.001$.

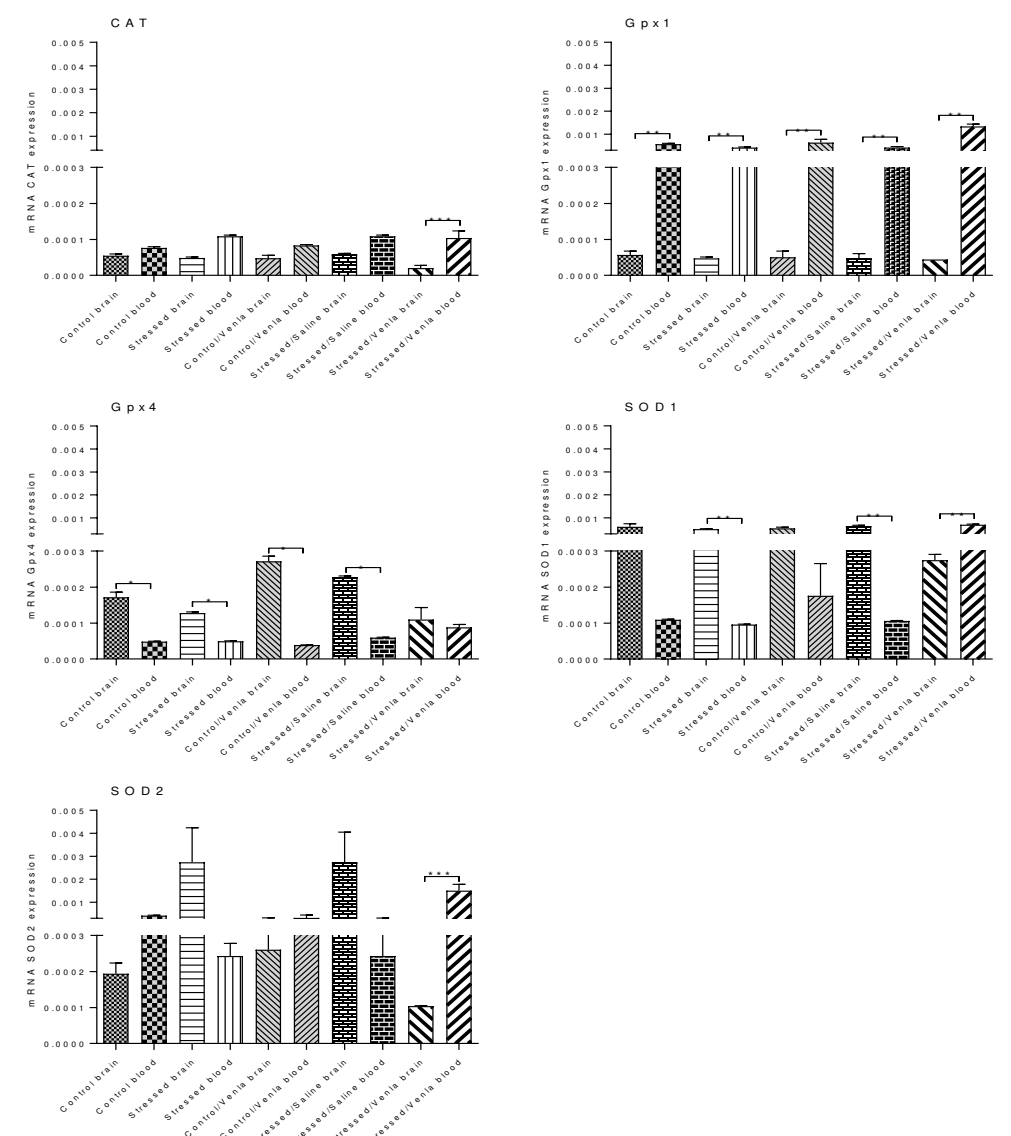

Differences in CAT, Gpx1, Gpx4, SOD1 and SOD2 gene expression between brain tissue and PBMCs of animals exposed to CMS for two weeks (Control, Stressed) and in animals exposed to CMS for seven weeks and administered vehicle $(1 \mathrm{~m} / \mathrm{kg})$ or venlafaxine $(10 \mathrm{mg} / \mathrm{kg})$ for five weeks (Control/Venla, Stressed/Saline, Stressed/Venla). The mRNA relative expression levels of all 5 genes measured in hippocampus, amygdala, hypothalamus, midbrain, cortex and basal ganglia estimated using a $2-\Delta \mathrm{Ct}(\mathrm{Ct}$ gene-Ct $18 \mathrm{~S})$ method. Data represent means $\pm \mathrm{SEM} . \mathrm{N}=6 .{ }^{*} p<0.05$, ${ }^{* *} p<0.01,{ }^{* * *} p<0.001$ 